\title{
Experiencias de innovación en la práctica docente en educación superior. De la reflexión a la acción
}

Dra. Rosamary Selene Lara Villanueva, Mexicana.

https://orcid.org/0000-0001-7139-2062

rosamary@uaeh.edu.mx

Dra. Maricela Zúñiga Rodríguez, Mexicana.

https://orcid.org/0000-0002-8055-3742

innomary2014@gmail.com

Universidad Autónoma del Estado de Hidalgo, México.

Recibido: 24 de junio del 2020

Aceptado: 25 de agosto del 2020

\section{Resumen}

Actualmente la innovación en la práctica docente es un tema relevante en las instituciones de educación superior para fortalecer las competencias y el ejercicio profesional docente así como para lograr mejoras notables en los procesos de enseñanza y aprendizaje, mismas que se concretan en experiencias docentes mediante proyectos de innovación en universidades públicas y en escuelas normalistas como es el caso de la Red de Comunidades para la Renovación de la EnseñanzaAprendizaje en Educación Superior (RECREA). En el presente artículo se describe y analizan las experiencias de innovación de la práctica educativas de docentes de la Universidad Autónoma del Estado de Hidalgo llevadas a cabo en el proyecto RECREA durante los procesos de formación y los diseños instruccionales que se derivaron de esta, así como la implementación de la experiencia en el aula en el año 2018, que fue el primer año del proyecto. La metodología para analizar estas experiencias se basó en la investigación-acción porque a partir de la reflexión-acción, los docentes pueden mejorar sus prácticas. Por tanto, la participación y la colaboración tienen un papel importante en los procesos de innovación. Con la descripción de las etapas de formación durante el proyecto RECREA, se pudo obtener información relevante para analizar los procesos de mejora como de oportunidades para cambiar las prácticas docentes. Los ejes transversales desarrollados durante el proyecto para la transformación de la práctica docente que se integran en la reflexión son: i) Pensamiento complejo, ii) Uso de las TIC e iii) Investigación-acción, que sirvió como metodología para abordar el proceso de innovación en la práctica docente. Los resultados encontrados, indican que hubo una mejoría sustancial en la práctica docente y en el aprendizaje de 
los estudiantes. También, motivó al diseño de estrategias más creativas y menos fragmentadas como se ha acostumbrado. Entonces, como todo proceso de mejora, la reflexión-acción, hizo posible que los docentes replantearan sus prácticas de enseñanza y generaran conocimiento, como resultado de sus experiencias educativas.

Palabras claves. Reflexión-acción, pensamiento complejo, innovación, práctica docente, educación superior

\title{
Innovation experiences in higher education teaching practice. From reflection to action
}

\begin{abstract}
At current time, innovation in teaching practice is a relevant issue in higher education institutions to strengthen teaching skills and professional practice as well as to achieve notable improvements in teaching and learning processes, which are materialized in teaching experiences through educational and innovative projects in public universities and Normal schools. This is the case of the Communities Network for the Renewal of Teaching-Learning in Higher Education, called RECREA project in spanish. This paper analyzes the innovation experiences in the teaching practice of the Autonomous University of the Hidalgo State in the RECREA project during the training processes and the instructional designs that were derived from it, as well as the implementation of the experience in classrooms during 2018, which was the first year of the project operation. The methodology to analyze these experiences was based on action research because with it teachers can improve their practices from action-reflection. Therefore, participation and collaboration play an important role in innovation processes. With the description of the training stages during the RECREA project, it was possible to get relevant information in order to analyze the improvement processes, as well to identify opportunities to modify these teaching practices. The results show that there was a substantial improvement in teaching practices and in student learning. It also motivated the design of more creative and less fragmented strategies as it was a customary practice. So, like any improvement process, reflection-action made it possible for teachers to rethink their teaching practices and generate knowledge as a result of their educational experiences.
\end{abstract}

Keywords: Reflection-action, complex thinking, innovation, teaching practice, higher education 


\section{Introducción}

Ante la presencia y la exigencia de enfoques de formación y de un nuevo perfil docente en educación superior, se considera que ésta debe estar orientada hacia modelos de desarrollo profesional, donde prevalezca la reflexión de la acción. El docente será un profesional que esté comprometido con su práctica y con la responsabilidad social de ejercer sus funciones con autonomía, ética, compromiso y que reflexione para mejorar.

En este sentido, el perfil profesional del docente de educación superior de este siglo, debe poseer competencias que estén en constante actualización que involucre la importancia de la tecnología como un medio y una herramienta eficaz para aplicar en clases, y que los estudiantes la utilicen de la forma más eficiente para aprender (UNESCO, 2008). Con el uso de las TIC se pueden desarrollar nuevas formas de enseñanza adecuando las metodologías al tipo o características de los estudiantes, al contexto y la naturaleza de la disciplina profesional; para ello, se requiere de competencias profesionales que se adecuen a las exigencias propias de la sociedad actual y a los avances científicos y tecnológicos con la finalidad de incrementar la calidad en la educación superior.

En las instituciones de educación superior, los docentes que ingresan a impartir alguna asignatura tienen poca preparación pedagógica para enseñar los conocimientos disciplinares y es durante el desarrollo de su práctica docente que empiezan a adquirir las habilidades para enseñar, si bien algunos se forman de manera particular, las instituciones educativas en su mayoría, son las encargadas de formarlos, o bien capacitarlos en competencias pedagógicas, tecnológicas y metodológicas para impartir sus asignaturas. Como sucede con la Universidad Autónoma del Estado de Hidalgo, que ofrece capacitación a sus docentes.

Por otra parte, también se dan otros procesos de formación docente a partir de la participación de proyectos académicos que se desarrollan en redes de investigación como es el caso concreto de la la Red de Comunidades para la Renovación de la Enseñanza-Aprendizaje en Educación Superior en sus siglas RECREA (SEP, 2017), en el cual participaron docentes miembros de los Cuerpos Académicos del Área Académica de Ciencias de la Educación de la UAEH. 
Las consideraciones anteriores ponen de relieve la preocupación por mantener la calidad educativa para involucrar a los docentes en la investigación educativa orientada a mejorar la práctica docente (Chacón, 2013). A su vez, Schoonmaker (2007), citado por Muñoz \& Garay (2015), señalan que propuestas alternativas como la formación profesional continua y las comunidades de aprendizaje, tienen un papel relevante en la transformación de la escuela mediante la inclusión de la investigación, lo que constituye una ventaja significativa cuando se establece la conexión entre la investigación y la práctica.

Muñoz y Garay (2015), Sánchez y Escamilla (2018). comparten este aspecto cuando señalan que existen iniciativas exitosas a nivel nacional como internacional que se pueden ver en diferentes instituciones de educación superior., lo que posibilita que los docentes como investigadores difundan este nuevo conocimiento y conozcan sus pares académicos.

Un elemento clave del proyecto RECREA fue que los docentes documentaran sus experiencias educativas y resultados como parte de todo un proceso de mejora en sus prácticas. El presente artículo, da cuenta de ello a través del análisis de las experiencias de inovación de la

práctica docente, a partir de las publicaciones realizadas en el proyecto, así como de los procesos de reflexión acción durante el diseño instruccional y su implementación en el aula.

La investigación-acción como metotodología fue un referente importante para abordar el proceso de innovación en la práctica docente.

\section{La innovación en la práctica docente en educación superior}

En las instituciones de educación superior, un tema bastante recurrente es cómo lograr la anhelada calidad educativa y específicamente en los procesos de enseñanza-aprendizaje y ello apunta inevitablemente al papel que el docente tiene en este aspecto.

Se desarrollan políticas y programas como PRODEP (SEP, 2020) para formar a los docentes y se estimula esta participación con becas o compensaciones económicas para que se produzcan los cambios institucionales como es el Programa del Estímulo a la docencia; sin embargo, parece que en el terreno de lo práctico, es decir, lo que hacen los docentes y estudiantes en el aula, se motiva poco el seguimiento para la innovación de la práctica docente, el trabajo 
colaborativo o colegiado se realiza muy poco y prevalece el trabajo individual. Este cambio puede ocurrir si los líderes o autoridades planifican estrategias de autorreflexión, evaluación de las prácticas en espacios cercanos al docente y se establezcan diálogos para mejorar de forma efectiva la docencia.

Por ello, recuperar las vivencias compartidas de los docentes en distintos escenarios para el intercambio de experiencias educativas en las instituciones de educación superior, promueve el desarrollo de ideas colectivas para realizar proyectos investigación y transformar las prácticas de los docentes. Las academias institucionales en los programas educativos que se trabajan en la Universidad Autónoma del Estado de Hidalgo (UAEH, 2019), constituyen excelentes espacios de reflexión, para hacer recomendaciones y propuestas educativas que pueden ser potenciales para desarrollar innovaciones en las prácticas de los docentes.

Los escenarios de aprendizaje acercan a los docentes a conocer paradigmas centrados en el aprendizaje, alcanzar las innovaciones que deben desarrollarse e introducirse en los pensamientos, en la organización, en la política educativa y en las prácticas pedagógicas (López, 2017). Ello, exige cuidar la coherencia lógica y ética del discurso político educativo con las prácticas docentes que se realizan en las instituciones de educación superior. Del mismo modo, dicho autor advierte que la innovación en la práctica docente de educación superior, no significa necesariamente un cambio, aunque puede calificarse como innovación, debe implicar un proceso razonado para avanzar hacia la mejora del conocimiento.

La práctica docente es una labor profesional intencional y deliberada que se lleva a cabo dentro y fuera del aula para provocar el aprendizaje en los estudiantes y este queda identificado con los procesos, pero también con las personas y con las actitudes intencionadas que provocan transformaciones socioeducativas. Un aspecto en la innovación según Zabalza (2013), es el significado que se le otorga porque no sólo implica hacer cosas distintas, sino que ocurra una mejora o mejoras de forma sustancial, tanto en el plano personal, como social, para que transforme las acciones y las sinergias del docente para innovar. La tarea en este proceso de innovación docente requiere de una participación colaborativa y al mismo tiempo de una introspección en el plano individual para que se observen las mejoras sustanciales que señala dicho autor.

Revista RedCA octubre 2020 - enero 2021 ISSN: 2594-2824 Vol. 3 Núm. 8 


\section{La reflexión, como praxis en la formación de comunidades de práctica}

La reflexión en la acción como epistemología más adecuada para el estudio de las actividades de la docencia establece una importante categoría de análisis para estudiar las acciones que realizan los profesionales de la enseñanza y los resultados que se derivan de ellas para mejorar las prácticas docentes. Esta delimitación en la relación teórica-práctica, como praxis de formación se construye en el proceso de intervención directa por parte del docente para mejorar e innovar (Pedroza, Villalobos \& Nava, 2014).

De esta manera, un profesional de la docencia que reflexiona en la acción y construye sus propias categorías de acción, formas de búsqueda y teorías aprende y definir situaciones problemáticas, actúa, incorpora y trasciende el conocimiento rutinario reglado por la racionalidad técnica, para construir nuevas estrategias de acción e innovar sus experiencias educativas (Latorre, 2013), lo que significa, que estén en constante revisión y se genere la autocrítica que permita explicar sus acciones y reorientar sus prácticas pedagógicas, como señala Castellanos \& Yaya (2013), mirar la práctica docente de forma compleja, es un punto de partida para una acción más rica, menos fragmentada y que implica un pensamiento reflexivo.

Con el proceso de reflexión-acción, el docente puede tener la oportunidad de confrontar de forma empírica sus esquemas teóricos y su realidad, siendo un primer espacio para replantear la realidad problemática para refutar sus planteamientos previos y que pueda corregir, modelar y depurar (Gimeno Sacristán 2008). A su vez, Espejo (2012), menciona que la complejidad de la práctica educativa hace necesario que el docente asuma el papel de investigador, reflexione sobre múltiples situaciones a partir de la experiencia y que como actor y productor principal genere cambios en su trabajo.

La Complejidad y El Pensamiento Complejo dentro del proceso de enseñanza y aprendizaje

Revista RedCA octubre 2020 - enero 2021

ISSN: 2594-2824 Vol. 3 Núm. 8 
El concepto de la Complejidad es un concepto que nos acerca a mirar las realidades educativas desde lo múltiple, lo ambiguo, presenta una paradoja de uno y lo múltiple, representa un tejido de constituyentes heterogéneos inseparablemente asociados de nuestro mundo fenoménico, en donde aparece la contradicción, la incertidumbre y las retroacciones y azares (Morín, 2011). De esta forma, no hay realidad que podamos reducir, no pueden comprenderse los procesos de enseñanzaaprendizaje desde un aspecto reduccionista, desde sólo un ámbito de la didáctica, de lo pedagógico o bien desde lo psicológico y social, incluso de un espacio sólo escolarizado, por ello es necesario estudiar las distintas situaciones, contextos o dimensiones en que se desarrollan dichos procesos que surgen en la práctica de la enseñanza.

Desde esta mirada, Ruíz y Torres (2016) señalan que el pensamiento complejo puede desarrollarlo toda persona ya que está provista de habilidades y capacidades para diferenciar lo imaginario con lo real, la fantasía, el orden y el caos, sin embargo, puede haber confusión y perderse en este mar de conocimientos e incluso desde lo ético y lo moral, pero que ayuda a analizar los distintos ángulos de la enseñanza. Las revisiones teóricas arriba mencionadas, dan sustento al presente trabajo. A continuación se explica el enfoque metodológico empleado y se describe la metodología de trabajo desarrollada.

\section{Objetivos generales}

Analizar las experiencias de la innovación de la práctica docente, a partir de las publicaciones generadas durante su participación en el proyecto RECREA, así como en los procesos de reflexión durante el diseño instruccional y su implementación en el aula.

\section{Metodología de investigación}

Para explicar cómo ocurren estos procesos de reflexión-acción, se señala el enfoque metodológico de la investigación-acción.

Según Kemmis y MacTaggart (1988), la investigación-acción como vertiente de la metodología cualitativa está orientada hacia el cambio educativo y se caracteriza entre otras cuestiones por ser un proceso que se construye desde y para la práctica, pretende mejorarla través de su trasformación, al mismo tiempo que procura comprenderla. La participación de los sujetos en la mejora de sus 
propias prácticas, exige una actuación grupal por la que los sujetos implicados colaboran coordinadamente en todas las fases del proceso de investigación.

Por ende, la investigación-acción, ocupa un lugar preponderante en el contexto de participación de los docentes cuando investigan su propia práctica docente y participan de ella, a través de procesos de reflexión y de una búsqueda de mejora para cambiar su enseñanza.

En este sentido, como señala Colmenares \& Piñero (2008).

"Las personas implicadas directamente en la realidad objeto de estudio son también investigadores; los profesores son docentes, pero implica que ellos también son investigadores que exploran la realidad en que se desenvuelven profesionalmente. Queda atrás el docente "objeto" de estudio, ahora es el agente, el que decide y toma decisiones. En la investigación acción pueden participar los expertos (teóricos, investigadores, profesores de Universidad) como asesores o colaboradores, pero no son imprescindibles; sí lo son, en cambio, los implicados" (p. 106)

Al ser una metodología de investigación requiere de un rigor en su procedimiento, lo que implica la realización de tareas sistemáticas, basadas en la recoleccion de evidencias producto de la experiencia vivida por los docentes o actores que participan en el proceso de reflexión y de cambio (Colmenares y Piñero, 2008).

Criterios de selección de participantes: Ser miembro de los Cuerpos Académicos del Área Académica de Ciencias de la Educación y específicamente formar parte del Cuerpo Académico de Evaluación, Planeación y Desarrollo Curricular.

Participantes: 6 docentes de educación superior del Área Académica de Ciencias de la Educación. Universidad Autónoma del Estado de Hidalgo (UAEH), México

Asignaturas:Tres asignaturas de la Licenciatura en Ciencias de la Educación y dos de posgrado de la Especialidad en Docencia y Maestría en Ciencias de la Educación:

a) Licenciatura en Ciencias de la Educación:

Orientación Educativa y Tutoría donde se empleó la estrategia enseñanza situadaidentificación de problemas reales, (Veytia, Lara, \& Martínez, 2018).

Revista RedCA octubre 2020 - enero 2021 ISSN: 2594-2824 Vol. 3 Núm. 8 
Didáctica general, estrategia con vinculación teórico-práctica en la evaluación de la didáctica, (Melecio, Martínez, Lara \& Zamora, 2018).

Taller de investigación Educativa, estrategia con objetos virtuales de aprendizaje y trabajo colaborativo, (García, Veytia, \& Martínez, 2018).

b) Posgrado:

Contextos en los procesos de enseñanza y aprendizaje de la Maestría en Ciencias de la Educación se desarrolló como seminario-taller, (Lara, Rodríguez, Pérez y De Fuentes, 2018).

Metodología de la investigación de la Especialidad en Docencia se desarrolló como seminario y asesorías grupales, (Cáceres, Pérez, Martínez, \& García, 2019).

\section{Mecanismos de recolección de datos:}

- Revisión del proyecto RECREA durante los procesos de la formación docente.

- Revisión documental de las publicaciones generadas por los docentes participantes del proyecto RECREA y entrevista estructurada.

- Entrevista estructurada: Tuvo como propósito conocer cómo se llevaron a cabo los procesos de reflexión y el desarrollo de estrategias utilzadas en el diseño instruccional y su implementación en el aula. Se plantearon preguntas de reflexión a los docentes a través de una entrevista estructurada. Se desarrollaron tres preguntas que se enviaron por correo electrónico y las respuestas se recogieron por el mismo medio. La preguntas fueron:

1.A partir de la experiencia de realizar de forma conjunta el diseño instruccional con los elementos clave del proyecto RECREA ¿qué aprendí?

2. A partir de la experiencia de implementar el diseño instruccional en el aula, ¿qué aspectos se mejoraron en el aprendizaje de mis alumnos?

3. ¿Qué necesito para mejorar mi enseñanza y qué necesito mejorar en el aprendizaje de mis alumnos? 


\section{Procedimiento}

Para analizar cómo desarrollaron las experiencias educativas de los docentes, se procedió a organizar la información en 4 etapas, en las cuales se describieron primeramente los diferentes momentos metodológicos: 1) El proceso de formación para la integración de las comunidades de práctica, 2) La construcción de sus diseños instruccionales de acuerdo a la metodología didáctica del proyecto RECREA, 3) Desarrollo e implementación del diseño instruccional en el aula, 4) Documentación de la Experiencia educativa. También se llevó a cabo la entrevista que se estructuró en tres preguntas de acuerdo a: los aprendizajes obtenidos en el diseño instrucciónal, la implementación en el aula y, la valoración de su enseñanza y el aprendizaje de sus estudiantes.

\section{Descripción de las etapas}

\section{Primera etapa. El proceso de formación docente para la integración de las comunidades de práctica}

En 2018, el proyecto inició con la formación e integración de las comunidades de práctica de los académicos de Universidades Públicas Estatales y de Escuelas Normales y se llevaron a cabo las acciones de diseño instruccional e implementación en las aulas, las cuales se documentaron como experiencias educativas de los docentes en la UAEH durante los trabajos de RECREA el primer año.

1. Taller de conformación de Comunidades de Práctica (CoP), formación de los participantes en los lineamientos para la innovación de la práctica y diseño de un curso a impartir en el periodo académico inmediato.

Los trabajos se iniciaron a través de reuniones y talleres para explicar el proceso en un nuevo enfoque epistemológico del pensamiento complejo en la práctica docente. Se invitaron a docentes de Escuela Normal Valle del Mezquital, Centro Regional de Educación Normal, y de la Universidad Autónoma del Estado de Hidalgo, en específico de los Cuerpos Académicos del Área Académica de Ciencias de la Educación, con perfil Profesor de Tiempo Completo (PTC). Se explicó el procedimiento a seguir para trabajar con los elementos claves del diseño instruccional de forma colectiva como: a) Tareas/proyectos que refieren situaciones o problemáticas reales. b) 
Situaciones reales de orden creciente de complejidad, c) Identificación de saberes e información para lograr el desarrollo de competencias, d) Trabajar con un enfoque de pensamiento complejo y, e) Incorporar apoyos y modelamiento en la resolución de problemas para lograr el aprendizaje y la autonomía de los estudiantes.

\section{Segunda etapa:. La construcción de los diseños instruccionales de acuerdo a la metodología didáctica del proyecto RECREA}

Los diseños instruccionales se realizaron con base. en los seis pasos propuestos en el Proyecto RECREA, mismo que se retoma del Aprendizaje Complejo de Van Merriënboer y Kirschner (2013) y recogido en el documento Pasos para el diseño de tareas/proyectos de la Universidad Veracruzana (Proyecto Aula, 2010).

Estructura del diseño instruccional a partir de los seis pasos propuestos.

- Paso uno: contexto de la EE, unidad de competencia y subcompetencias.

1. Enuncie el perfil de egreso del programa educativo.

2. Describa brevemente cómo aporta esta EE al logro del perfil del egresado y su relación con otras EE del plan de estudios.

3. Reformule el propósito de la EE en términos de competencia o Unidad de competencia.

- Paso dos: tareas/ proyectos de aprendizajes, clases de tareas, objetivos de desempeño.

1. Enunciado de las "Tareas/ proyectos de aprendizaje" para la EE.

2. Definición y redacción de al menos una "Tarea/ proyecto de aprendizaje" con dos niveles de dificultad (clases de tareas) y las diferentes estrategias para desarrollar un pensamiento complejo, su vínculo con la investigación, y el uso de TIC, de acuerdo al nivel de dificultad.

Revista RedCA octubre 2020 - enero 2021 ISSN: 2594-2824 Vol. 3 Núm. 8 
3. Redacción de los objetivos de desempeño por cada clase o tipo de "tarea/ proyecto de Aprendizaje".

- Paso tres: información de apoyo (teórica y estratégica), procedimental y práctica de parte de las tareas.

- Paso cuatro: andamiaje de la tarea/ proyecto de aprendizaje

1. Para cada clase de tareas/ proyectos de aprendizaje de su EE, defina y redacte los siguientes elementos:

- Enunciado de la o las tarea(s)/ proyecto(s) de aprendizaje específico(s) para su EE en el siguiente ciclo académico. Las tareas específicas se diseñan o se les cambian datos o apoyos cada vez que se imparte la EE.

- Las actividades que llevarán a cabo sus alumnos y los apoyos que usted les proporcionará para cada actividad.

- Los productos resultantes de las actividades realizadas por los alumnos. Las fechas de entrega cambian cada vez que se imparte la EE.

- Paso cinco: evaluación de la resolución/ ejecución de la tarea/ proyecto de Aprendizaje

1. Para cada producto, enuncie los elementos y los criterios de desempeño en una rúbrica o escala de evaluación de al menos tres niveles de desempeño y tres elementos de observación.

2. Diga si dará retroalimentación a los alumnos en la evaluación intermedia o final.

3. Describa los criterios para integrar la calificación para el alumno sobre su desempeño en la tarea de aprendizaje.

- Paso seis: Presentación a los estudiantes de la(s) tarea(s)/ proyecto(s) de aprendizaje.

- La presentación debe ser atractiva y estructurada de forma que para los estudiantes sea claro lo que tienen que hacer a lo largo del curso. 
Se consideraron dentro de esta estructura, los tres ejes transversales propuestos en la metodología del proyecto RECREA: Desarrollo del pensamiento complejo, vinculación del aprendizaje con la investigación e incorporación del uso de las Tecnologías de la Información y la Comunicación. Los criterios a considerar para la construcción de las asignaturas fueron: La naturaleza de la asignatura, teórica, práctica o ambas, el número del semestre, la periodicidad de la unidad o unidades temáticas, el perfil de egreso, las competencias de salida de licenciatura y de posgrado.

Asimismo, se planificaron reuniones de trabajo para conocer los avances de los docentes, lo que permitió procesos de reflexión, comprensión y un acompañamiento pedagógico.

\section{Tercera etapa: Desarrollo e implementación del diseño instruccional en el aula}

En cada asignatura y taller implementado, los docentes realizaron un diagnóstico a través de preguntas y análisis para conocer los conocimientos previos de los estudiantes sobre los temas. Se presentó a los estudiantes la planeación del diseño instruccional, las actividades, el tiempo, los materiales de apoyo, las estrategias, la evaluación del proceso y los instrumentos de evaluación.

Se aplicaron diferentes estrategias para el desarrollo de habilidades de investigación, empleo de las TIC y actividades de pensamiento complejo, a través de lecturas crítica de textos para el desarrollo de la comprensión lectora, capacidad sintética y analítica, favorecer un pensamiento reflexivo a partir de situaciones problemáticas, capacidad para tomar decisiones, trabajo colaborativo, caracterización del contexto, sistematización de información con el uso de organizadores gráficos de programas digitales, creatividad en el uso de las TIC, entre otros.

En la evaluación, los docentes emplearon diversas formas para evaluar holísticamente el trabajo de los estudiantes, enfocados en el aspecto formativo como sumativo. En el caso de la maestría en ciencias de la educación se utilizó el Google Drive para colocar una e-portafolio. Se crearon rúbricas para evaluar todo el proceso como cada criterio de la producción generada. En las asignaturas de la licenciatura en ciencias de la educación: Taller de investigación educativa, se utilizó el Objeto Virtual de Aprendizaje; en la de Tutoría y Orientación educativa se evaluaron tres dimensiones: metacognitivas, de procesamiento de la información y de control de contexto. En la asignatura Didáctica se instrumentó una rúbrica para evaluar la aplicabilidad de un diseño de 
evaluación para la planeación y en la asignatura de Metodología de la investigación de la Especialidad en Docencia, se dio seguimiento a los proyectos terminal de titulación de los estudiantes, mediante la problematización de la práctica docente, se abordó como un ejercicio heurístico desde la parte teórica, como metodológica.

\section{Cuarta etapa. Documentación de la experiencia educativa}

Derivado de las experiencias que los docentes adquirieron durante su formación, se revisaron las publicaciones de las experiencias educativas de los docentes, lo que les permitió difundir los resultados de sus propuestas en su diseño instruccional y su implementación. Esta revisión documental permitió hacer un análisis de los resultados obtenidos de cada docente participante.

Durante la revisión se encontraron diferentes formar de plantear las estrategias de enseñanza, de intregar las TIC y de alcanzar los objetivos de aprendizajes que fomentaran el pensamiento complejo junto con la investigación. Se pudo observar el diseño de tareas y las secuencias de actividades, así como la valoración de las experiencias por parte de los docentes que trabajaron en pares académicos.

\section{Resultados del proceso de implementación}

Los resultados en el proceso de implementación de los diseños instruccionales confirmaron que los profesores mejoraron sustancialmente sus prácticas y se crearon propuestas innovadoras de aprendizaje, considerando la relevancia de los tres ejes transversales del proyecto.

En cuanto a los aprendizajes de los estudiantes, se observó que pasaron de procesos cognitivos básicos, a procesos de pensamientos cognitivos y sociales más complejos, incrementando el pensamiento crítico para analizar, argumentar, conectar y evaluar la información, al tiempo que estimuló el pensamiento creativo con el desarrollo de estrategias metacognitivas y el potencial de sus habilidades en el uso de herramientas digitales, lo que se evidenció en sus productos y actividades de aprendizaje. Los estudiantes aprendieron a desarrollar habilidades colaborativas porque se emplearon estrategias de trabajo en equipo. 
En un proceso de reflexión, los docentes señalaron la necesidad de mejorar sus estrategias de enseñanza porque en algunos momentos de la implementación (tareas y elaboración de productos) no lograron alcanzar los objetivos de desempeño esperados en sus estudiantes, esto significó volver a reflexionar en pares para replantear las estrategias de enseñanza y aprendizaje.

Los docentes consideraron ser coherentes con los objetivos, contenidos y con una secuencia didáctica clara e incorporar metodologías didácticas activas que potencien el aprendizaje colaborativo, el pensamiento crítico y creativo que permite alcanzar las competencias específicas y genéricas de los programas educativos. Estas experiencias educativas deben darse de forma constante en la formación del profesorado y generar una cultura de colaboración y de investigación para innovar.

\section{Resultados de la entrevista a los docentes. Procesos de reflexión y análisis}

A modo de resumen se mencionan algunas respuestas de los docentes.

En la primera pregunta: ¿A partir de la experiencia de realizar de forma conjunta el diseño instruccional con los elementos clave del proyecto RECREA ¿qué aprendí?:

"Conocí una versión diferente de planificar la asignatura que permitió enriquecerla aún más,"

"que se pueden articular el uso de TIC, el pensamiento complejo y la investigación docente en cada etapa de la planeación didáctica del seminario donde se implementó"

"reafirmar algunos procesos del diseño didáctico de una clase. "Revisar mi papel como docente, la importancia de dar instrucciones claras, retomar el trabajo colaborativo, desarrollo de más rúbricas, el uso de la investigación acción como una parte de la metodología para revisar y mejorar mis prácticas".

En la segunda pregunta: A partir de la experiencia de implementar el diseño instruccional en el aula, ¿qué aspectos se mejoraron en el aprendizaje de mis alumnos? respondieron que: 
"los estudiantes aprendieron a desarrollar habilidades para una planificación fincada en intereses propios".

También, se desarrollaron habilidades colaborativas, sin dejar el lado el trabajo autónomo, los estudiantes fueron protagonistas de su propio proceso.

"Con el trabajo grupal los alumnos se enriquecieron con el trabajo de investigación que cada alumno aportaba, capacidad de reflexión, de análisis, de escucha y de aportaciones sobre cada tema";

"Se mejoró la calidad del trabajo colaborativo, el fomento al trabajo autónomo, la reflexión, la crítica constructiva, así como la parte de la creatividad, la responsabilidad, y la empatía con sus compañeros”.

En la tercera pregunta ¿Qué necesito para mejorar mi enseñanza y qué necesito mejorar en el aprendizaje de mis alumnos? respondieron que:

"es necesario recuperar las experiencias de la aplicación y la diversidad de respuestas de los alumnos, con la finalidad de mejorar el diseño instruccional"

“Tener mayor dominio del proceso del paradigma de la complejidad, planear considerando los objetivos que no se lograron con la anterior planeación, aprender más sobre el uso de nuevas TIC, e incluir en la planeación las acciones de investigación acción"

"Disponer de mayor tiempo para preparar los materiales".

Dichas reflexiones, permitió a los docentes conocer sus prácticas de enseñanza y renovarlas. La colaboración y la motivación también fueron aspectos relevantes para crear las mejores estrategias y planificarlas cuidadosamente. Por consiguiente, se enriquecieron las propuestas. lo que dio lugar a innovar en su práctica y se promovió el diseño de estrategias más creativas y menos fragmentadas como se acostumbraba.

\section{Discusión}


Los hallazgos encontrados en las experiencias educativas llevadas a cabo por los docentes de la UAEH que participaron en el proyecto RECREA, reflejan diferencias en cuanto a las expectativas y significados encontrados; por un lado, señalan que el desarrollo de sus diseños instruccionales permitió conocer otra maneras distintas de realizar sus planeaciones didácticas, lo que les condujo a reorientar sus prácticas docentes. Por otro lado, se encontraron con dificultades para transformar sus diseños, ya que estaban acostumbrados a desarrollar su instrucción de una manera fragmentada, sin considerar los aspectos de la complejidad, la investigación y el uso de las TIC, en las tareas de aprendizaje propuestas.

Esta forma de trabajo en los diseños instruccionales creó incertidumbre y dudas durante la formación docente, porque aún cuando se llevaban a cabo reuniones de trabajo para dar seguimiento a los diseños instruccionales, sentían que no lograban avanzar y que necesitaban ejemplos especificos para estructurar sus planeaciones. El acompañamiento en la primera etapa de formación fue un aspecto que suscitó algunos desafíos importantes para esclarecer las dudas y dar seguimiento a los diseños. Sin embargo, en la medida en que los docentes avanzaban sucedían momentos de reflexión y de participación colaborativa para apoyarse como comunidades de práctica. Este fue un componente del proyecto RECREA, que ofreció las oportunidades para modificar las prácticas de trabajo para mejorar su enseñanza.

Con el trabajo colaborativo y las lecturas propuestas sobre estrategias de enseñanza y aprendizaje, se promovió el diseño de estrategias más creativas y menos fragmentadas como se acostumbraba. Por tanto, se comprende que la "acción es estrategia", según Morin, 2011, es imaginar que las situaciones de aprendizaje pueden darse en diferentes escenarios para la acción, incluso estos podrían ser modificados y que las estrategias debían suponer la noción de ecología de la acción es decir, que la acción supone complejidad porque hay elementos, aleatorios, decisiones, transformaciones que, en algún punto o situación puesta en marcha en el diseño instruccional (planificación), haría volver como un boomerang para tratar de corregir el diseño y replantear de nuevo las tareas y los proyectos.

Además de dar oportunidades de cambiar las prácticas docentes y con ello replantear la enseñanza y el aprendizaje, los docentes intercambiaron sus experiencias, como pocas veces suceden cuando trabajan en solitario. Participar en proyectos de investigación reconforta el espíritu 
de ser autores y coautores de una experiencia educativa para darla a conocer a otros docentes de instituciones de educación superior, a través de la publicación de sus resultados. Por supuesto, es evidente que falta mucho por hacer para proponer innovaciones generadas desde la reflexión y con ello a una acción específica para mejorar, la investigación-accion representa un modo de analizar las prácticas docentes y de mejorar cada vez, por lo que se presenta como una herramienta epistémica orientada hacia el cambio educativo, como señalan Colmenares \& Piñeras (2008).

Por otra parte, la orientación de las tareas con el uso de las TIC, fue un aspecto que sobresalió, a pesar de que los docentes lo propusieron en sus diseños instruccionales. Tambien, se pudo observar que algunos docentes destacaron las habilidades de los estudiantes en el empleo de las TIC, como una ventaja que permitíó desarrollar sus tareas de forma creativa, al mismo tiempo que fortalecía las habilidades para la investigación, aspecto que se reflejó en los productos generados por los estudiantes..

Finalmente con la reflexión, los docentes pudieron percatarse de la importancia de dedicar más tiempo a la planeación didáctica, que requiere de un cuidadoso análisis para identificar dónde se encuentran las dificultades y fortalezas de sus estudiantes y generar estrategias de aprendizaje para promuever habilidades de pensamiento complejo, habilidades para la investigación y el uso de las TIC.

\section{Conclusiones}

La incertidumbre provocada durante el diseño, planificación, implementación y valoración de experiencias educativas es un efecto que conduce a fortalecer las habilidades de enseñanza de los docentes y conlleva invariablemente a replantear las prácticas, por ello es natural que se generen expectativas en y entre los docentes para lograr que el aprendizaje de los estudiantes sea superior al alcanzado en semestres anteriores, de ahí que las experiencias recogidas en este primer año de formación hacia la innovación con el proyecto RECREA, se reconozca el compromiso profesional para mejorar cada vez y brinde a los docentes las oportunidades para reconstruir las propias teorías acerca de cómo enseñar y cómo pueden aprender sus estudiantes durante el desarrollo de sus tareas.

En este sentido, la investigación-acción brindó a los docentes la oportunidad de transformar los conocimientos sobre sí mismos para teorizar y revisar sus experiencias. Sirve también para 
emprender actividades investigadoras y encaminar la formación docente hacia una cultura profesional que ayude a desarrollar la necesidad de conocimiento más profundo de la propia práctica, formular alternativas de acción y diagnosticar una situación a través de la reflexión.

También, un aspecto importante a destacar fue el intercambio de conocimientos y la reflexión entre docentes de instituciones de educación superior universitaria (UAEH), como de las escuelas normales.La interacción durante los avances de los diseños en reuniones de trabajo y seguimiento, propiciaron reflexiones y diálogos que redundaron en una alta motivación para continuar incrementando el conocimiento pedagógico obtenido a través de las innovaciones de la práctica docente. Esta forma de trabajo abrió oportunidades para compartir buenas prácticas entre profesores, evaluar las dificultades, crear soluciones y nuevas alternativas para facilitar y mejorar los aprendizajes de los alumnos. Cumpliéndose así, el objetivo para el cual fue creado RECREA, y por supuesto las innovaciones generadas por los docentes al diseñar sus tareas de aprendizaje y los procesos de cambio que ocurrieron en la implementación en sus aulas.

\section{Referencias bibliográficas}

Blanco, G. (2012). La innovación en la práctica docente. Graffylia Revista de la Facultad de Filosofía y Letras. Benemérita Universidad Autónoma de Puebla 14-15/10, 80-89. Recuperado de: https://filosofia.buap.mx/content/graffylia-no6

Cáceres, M., Pérez, C, Martínez, O. \& García, O. (2019). The renewal of the teaching and learning processes in the subject of methodology of the research in the specialization in teaching. International Journal of Development Research. 9(2), pp.26030-26036. Available online at http://www.journalijdr.com

Castellanos, Sonia, \& Yaya, R. (2013). La reflexión docente y la construcción de conocimiento: una experiencia desde la práctica. Sinéctica, (41), 2-18. Recuperado de: http://www.scielo.org.mx/scielo.php?script=sci arttext\&pid=S1665109X2013000200006\&lng=es\&tlng=es.

Chacón, J. (2013). Una experiencia en la enseñanza de la investigación educativa en el marco de "Proyecto Aula" de la Universidad Veracruzana. Revista Mexicana de Investigación Educativa, 18(58), 735-768. Recuperado de: http://www.scielo.org.mx/scielo.php?script=sci_arttext\&pid=S1405$\underline{66662013000300004 \& \operatorname{lng}=\mathrm{es} \& \mathrm{t} \operatorname{lng}=\mathrm{es} .}$

Colmenares E., \& Piñero, Ma. (2008). La investigación-acción. Una herramienta metodológica heurística para la comprensión y transformación de realidades y prácticas socioeducativas. Laurus, $14(27), 96-114$. de: https://www.redalyc.org/articulo.oa?id=761/76111892006

Recuperado

Revista RedCA octubre 2020 - enero 2021 ISSN: 2594-2824 Vol. 3 Núm. 8 
Espejo, R. (2010). Algunos aspectos de la educación compleja, Polis. 9(25), 1-14. Recuperado de http://journals.openedition.org/polis/322

García, O., Veytia, M.G. \& Martínez, O. (2018). Objetos virtuales de aprendizaje como apoyo en los procesos de renovación para el desarrollo de competencias para la investigación en un programa de educación superior. Ponencia presentada en IX Seminario Internacional de Docencia Universitaria, CEDDES. Cuba: Universidad e Cienfuegos, Disponible en: https://universosur.ucf.edu.cu/index.php/en/catalogo-de-publicaciones/item/145-ixseminario-internacional-de-docencia-universitaria

Gimeno Sacristán, J. (2002). Comprender y Transformar la enseñanza. Madrid: Morata.

Kemmis, S, \& MacTaggart, R. (1988). Cómo planificar la investigación-acción. Barcelona: Laertes.

Lara, R., Rodríguez, M. Pérez, C. \& De Fuentes, A. (2018). El aprendizaje complejo. Una experiencia educativa aplicada con estudiantes de posgrado en la Universidad Autónoma del Estado de Hidalgo (UAEH). México. Ponencia presentada en IX Seminario Internacional de Docencia CEDDES Universitaria. Cuba: Universidad e Cienfuegos, Disponible en: https://universosur.ucf.edu.cu/index.php/en/catalogo-depublicaciones/item/145-ix-seminario-internacional-de-docencia-universitaria

Latorre, A. (2013). Conocer y cambiar la práctica educativa. Graó: México.

López, R. (2017). Hacia una innovación docente de calidad en la educación superior, Claves para la reflexión. Foro Educacional. 8, 11-28. Recuperado de: https://dialnet.unirioja.es/servlet/articulo?codigo $=6429501$

Melecio, A, Martínez, O. Lara, R. \& Zamora, D. (2018). Una experiencia educativa desde la Didáctica General, Ponencia presentada en IX Seminario Internacional de Docencia CEDDES Universitaria. Cuba: Universidad de Cienfuegos, Disponible en: https://universosur.ucf.edu.cu/index.php/en/catalogo-de-publicaciones/item/145-ixseminario-internacional-de-docencia-universitaria

Morin, E. (2011). Introducción al Pensamiento Complejo. Barcelona: Gedisa

Muñoz, M, \& Garay, F. (2015). La investigación como forma de desarrollo profesional docente: Retos y perspectivas. Estudios pedagógicos (Valdivia), 41(2), 389-399. https://dx.doi.org/10.4067/S0718-07052015000200023

Pedroza, R., Villalobos, G. \& Nava, N. (2014). Un método para la práctica educativa y creativa. México: MAPorrúa, librero editor: Universidad Autónoma del Estado de México.

Proyecto Aula (2010). Pasos para el diseño de tareas / proyectos de Aprendizaje para el desarrollo de competencias y Pensamiento complejo. Universidad Veracruzana. Recuperado de: https://www.uv.mx/personal/joacosta/files/2010/07/4-Pasos-D.I.1.pdf

Revista RedCA octubre 2020 - enero 2021 ISSN: 2594-2824 Vol. 3 Núm. 8 
Ruíz, B. C. \& Torres, L. C. (2016). Pensamiento complejo: transformación del aprendizaje. Comunicación, cultura y política, 213-240. https://doi.org/10.21158/21451494.n0.2016.1770

Sánchez, M. y Escamilla, J. (2018). Perspectivas de la innovación Educativa en Universidades de México. Experiencias y reflexiones de la RIE360. México: Imagina. Recuperado de: https://www.amfem.edu.mx/phocadownload/publicaciones/Perspectivas_de_la_innovacio \%CC\%81n educativa en universidades de Me\%CC\%81xico.pdf

SEP (2017). Proyecto de Investigación de la Comunidades de Aprendizaje para la Renovación de los Procesos de enseñanza y Aprendizaje de la Educación Superior, México. Recuperado de: https://promepca.sep.gob.mx/solicitudesca/login.aspx

SEP (2020). Programa para el Desarrollo Profesional Docente, para el Tipo Superior (PRODEP) Subsecretaría de Educación Pública, México. Recuperado de: http://www.dgesu.ses.sep.gob.mx/PRODEP.htm

UAEH (2019). Reglamento de Academias. Universidad Autónoma del Estado de Hidalgo, Pachuca, Hidalgo, México. Recuperado de: https://www.uaeh.edu.mx/adminyserv/dir generales/juridica/pdf/2019/Reglamento-deacademias.pdf

UNESCO (2008). Estándares de Competencia en TIC para Docentes. Recuperado de http://www.eduteka.org/EstandaresDocentesUnesco.php

Van Merriënboer, J. G., \& Kirschner, P. (2013). Ten steps to complex learning: A systematic approach to four-component instructional design (2nd ed.). New York: Routledge

Veytia, M., Lara, R. \& Martínez, O. (Octubre, 2018). La formación tutorial en Educación Superior con empleo de las TIC. Ponencia presentada en IX Seminario Internacional de Docencia Universitaria. Cuba: CEDDES, Universidad de Cienfuegos. Recuperado de: https://universosur.ucf.edu.cu/index.php/en/catalogo-de-publicaciones/item/145-ixseminario-internacional-de-docencia-universitaria

Zabalza, M. (2013). Innovación en la enseñanza universitaria. Contextos Educativos. Revista de Educación, 0(6), 113-136. DOI: https://doi.org/10.18172/con.53

Revista RedCA octubre 2020 - enero 2021 ISSN: 2594-2824 Vol. 3 Núm. 8 